MAYO CLINIC ए5)

Background

Premature and low birth weight infants have been found to have delays and lapses in immunization that persist through early childhood.

Adherence to the routine immunization schedules such as the schedule recommended by the United States Advisory Committee for Immunization Practices (ACIP) minimizes the risk of contracting vaccine preventable illnesses in this vulnerable population.

We performed an audit of infant immunization rates for our 26bed neonatal intensive care unit to evaluate this measure. We used quality improvement methodology to identify root causes for under immunization and guide improvement.

\section{Objective}

We sought to increase the percentage of infants fully upto-date for routine immunizations at the time discharge or unit transfer from the Mayo Clinic NICU from a baseline of $56 \%$ to a goal of $80 \%$ by March 31, 2018.

\title{
Improving On-Time Immunization Rates in the Mayo Clinic Neonatal Intensive Care Unit
}

Raymond Stetson, M.D., ${ }^{1}$ Jennifer Fang, M.D., M.S., ${ }^{1}$ Christopher Colby, M.D. ${ }^{1}$ and Robert M. Jacobson, M.D. ${ }^{2}$ 1 Division of Neonatal Medicine, 2 Divisions of Community Pediatric \& Adolescent Medicine and Pediatric Infectious Diseases

Mayo Clinic, Rochester, Minnesota

\section{Methods}

Electronic health records (EHR) were reviewed to obtain baseline immunization rates for all infants discharged or transferred from the Mayo Clinic NICU, Rochester, MN from January 1 2015 to June 30, 2017. Infants were counted as fully up-to-date if they had received all immunizations due per the ACIP immunization schedule, with the exception of rotavirus vaccination.

Immunization rates were similarly ascertained for all infants with monthly audits during the study period from October 1, 2017 through March 31 2018.

Using DMAIC-Define, Measure, Analyze, Improve, Contro/-quality improvement methodology, we performed root cause analysis of the under immunization rate. We analyzed baseline data including NICU provider $(n=37)$ and nursing surveys $(n=47)$ using a Pareto Chart, the 5 Whys, and a Fishbone diagram.

We identified three major root causes of the quality gap. These included lack of provider knowledge of the routine immunization schedule, providers not ordering vaccines when they were due, and parental vaccine hesitancy.

In the first plan-do-study-act (PDSA) cycle, the unit initiated three improvement measuresproviding intranet resources for NICU providers on the routine immunization schedule, education on vaccine hesitancy, and an Excel-based checklist to track when immunizations were due. The second PDSA cycle, the unit implemented improvements in parental vaccine consent documentation.

\section{Results}

The EHR of 912 infants were audited during the baseline period. Similar audits were performed for 171 infants during the six month intervention period

One-hundred sixty infants were excluded from the baseline audit because of death $(n=33)$ or unit transfer $(n=127)$ prior to any immunizations being due. Per the same exclusion criteria, we excluded 16 infants because of death $(n=5)$ or unit transfer $(n=11)$ during the study period.

During the baseline period, only $56 \%$ (419 of 754) were fully up-to-date at discharge or transfer compared to $94 \%$ (145 of 155) during the study period, p-value $<0.0001$. The run chart demonstrated significant process improvement following the two PDSA cycles (figure 1), with immunization rates exceeding the original goal of $80 \%$.

Figure 1: Run Chart

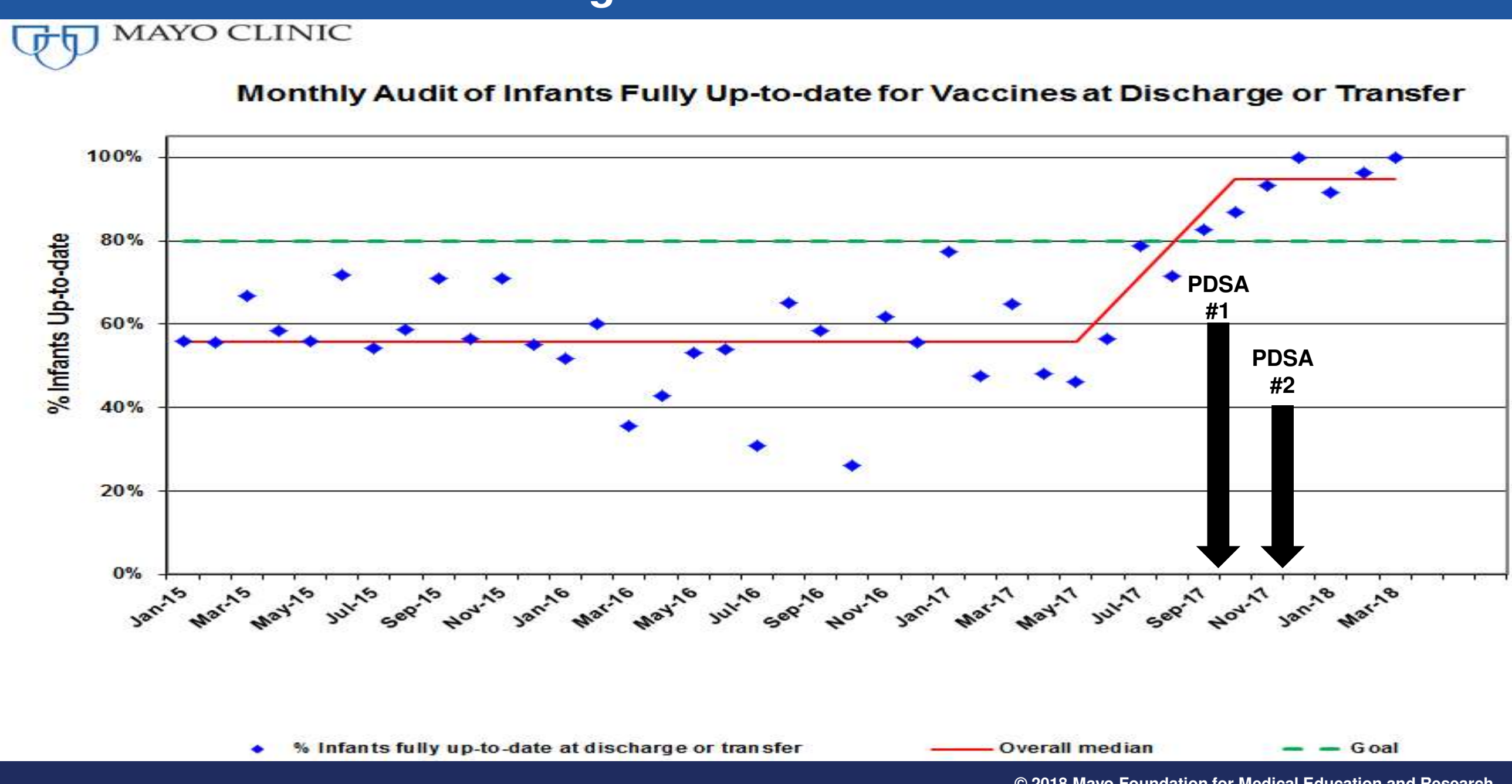

